\title{
Orbital parameters of merging dark matter halos
}

\author{
S. Khochfar ${ }^{1}$ and A. Burkert ${ }^{2}$ \\ 1 Department of Physics, Denys Wilkinson Building, Keble Road, Oxford OX1 3RH, UK \\ e-mail: sadeghk@astro.ox.ac.uk \\ 2 University Observatory Munich, Schreinerstr. 1, 81679 Munich, Germany
}

Received 14 April 2005 / Accepted 6 September 2005

\begin{abstract}
In order to specify cosmologically motivated initial conditions for major galaxy mergers (mass ratios $\leq 4: 1$ ) that are supposed to explain the formation of elliptical galaxies we study the orbital parameters of major mergers of cold dark matter halos using a high-resolution cosmological simulation. Almost half of all encounters are nearly parabolic with eccentricities $e \approx 1$ and no correlations between the halo spin planes or the orbital planes. The pericentric argument $\omega$ shows no correlation with the other orbital parameters and is distributed randomly. In addition we find that $50 \%$ of typical pericenter distances are larger than half the halo's virial radii which is much larger than typically assumed in numerical simulations of galaxy mergers. In contrast to the usual assumption made in semi-analytic models of galaxy formation the circularities of major mergers are found to be not randomly distributed but to peak around a value of $\epsilon \approx 0.5$. Additionally all results are independent of the minimum progenitor mass and major merger definitions (i.e. mass ratios $\leq 4: 1 ; 3: 1 ; 2: 1$ ).
\end{abstract}

Key words. methods: $N$-body simulations - cosmology: dark matter - galaxies: interactions

\section{Introduction}

Toomre \& Toomre (1972, TT72) proposed that elliptical galaxies could form from mergers of disk galaxies. Thereafter this "merger hypothesis" has been investigated in great details by many authors (for recent reviews see Burkert \& Naab 2003a,b) using numerical simulations. The first fully self-consistent models of two equal-mass stellar disks embedded in dark matter halos were performed by Negroponte \& White (1983), Barnes (1988) and Hernquist (1992). Typically, the spiral galaxies were constructed in dynamical equilibrium (Hernquist 1993a) and approached each other on parabolic orbits with pericenter distances of twice the scale length of the larger disk galaxy. Already the first simulations demonstrated that mergers lead to slowly rotating, pressure supported and anisotropic systems that generally follow a de Vaucouleurs surface density profile and resemble elliptical galaxies. Hernquist (1993b) noted that a massive central bulge component was required in the progenitors in order to explain the high stellar densities observed in the cores of ellipticals. More recent investigations showed that the initial mass ratio of the merging spirals determines the isophotal shapes and kinematical properties of the merger remnants (Bendo \& Barnes 2000) and it was suggested e.g. by Naab et al. (1999) that the initial mass ratio might explain the observed dichotomy between disky, fast rotating and boxy, slowly rotating ellipticals (Bender et al. 1988; Bender 1988).
Recently, Naab \& Burkert (2003) performed the to date largest parameter survey of collisionless binary disk mergers with varying mass ratios, different initial disk orientations and orbital parameters. They found that the fine structure of the merger remnant in general depends strongly on the adopted initial conditions. Certain initial conditions in fact lead to disky, anisotropic remnants which are not observed. Clearly, a deeper understanding of the origin of the early type family of galaxies not only requires a more sophisticated treatment of the various physical processes but also a better understanding of the initial orbital configurations of the interacting galaxies as predicted by currently favoured cosmological models.

Up to now due to the lack of knowledge of the appropriate initial conditions, simulations were forced to cover a wide parameter space by setting up mergers with different orbital configurations. This approach also has the drawback that it is not clear how relevant a given parameter combination is which leads to a certain fine structure of the merger remnants. In this paper we therefore present a detailed analysis of a high-resolution, large-scale cosmological simulation that was carried out by the Virgo Supercomputing Consortium using computers based at the Computing Centre of the Max-Planck Society in Garching and at the Edinburgh Parallel Computing Centre ${ }^{1}$. and derive the typical orbital parameters predicted by cosmology for merging dark matter halos which should also be representative for their visible galactic components.

\footnotetext{
${ }^{1}$ www.mpa-garching.mpg.de/NumCos
} 


\subsection{The cosmological simulation}

The simulation we analyse was performed within the GIF project (Kauffmann et al. 1999) and we will here focus on the $\Lambda \mathrm{CDM}$ simulation. This simulation was carried out in a box of size $141.3 \mathrm{Mpc} h^{-1}$ with $256^{3}$ particles each having a mass of $1.4 \times 10^{10} M_{\odot} h^{-1}$ and cosmological parameters $\Omega_{\Lambda}=0.7, \Omega_{0}=0.3, \sigma_{8}=0.9, h=0.7$ and a force softening of $20 \mathrm{Kpc} / h$. The softening leads to an error of a few percent in the calculation of the orbital parameters. The positions and velocities of the particles have been saved at 44 different redshifts. Additionally, for each output redshift, a list of halo properties have been provided by the GIF project. When two halos approach each other their orbit is going to change due to the transfer of orbital angular momentum to the halo's internal angular momentum which in the following is called spin and should not be confused with the cosmological spin parameter defined e.g. in Peebles (1993). The determination of the initial orbital parameters therefore is a question of the "right timing". We try to identify these parameters at a time when the interaction between the halos is still weak and one can assume a Keplerian two-body situation, using the positions of the most bound particles of each halo. At each redshift we go through the list of halos identified by using the friends-of-friends (FOF) algorithm and identify the positions of the most bound particles. If at one redshift a halo has disappeared through merging with another halo, we look up the position of its most bound particle at the previous redshift and check whether the distance to the most bound particle of the other halo, with which it is going to merge, is larger than the sum of both virial radii. If so, we derive the orbital informations using the data from this redshift. To make sure that the merger is not just a flyby of an unbound halo we check at a redshift later than the merging redshift whether the separation of the most bound particles has increased again. Since outputs are generated roughly every 0.5 Gyr we expect not to miss even very eccentric orbits.

\section{The reduced two-body problem}

The orbital parameters are defined as specified in the pioneering work of TT72. We simplify the problem by reducing the two-halo system to a two-body system with each halo being represented by its most bound particle which sits in the potential minimum and is expected to be the most "stable" particle, allowing us to follow the orbital evolution of the progenitor halos during the early stages of the merger even when a clear definition of the centre of mass of the extended and already partly overlapping progenitor halos is not possible anymore. We checked that the position of the most bound particle is not very different from the centre of mass of each halo when the halos are well separated and that it agrees with the region of highest dark matter density. We additionally tested if the results presented in this paper show any significant dependence on using the centre of mass of each halo, calculated by averaging the particle positions inside different fractions of the virial radius, instead of the position of the most bound particle and found no significant difference. The position of the most bound particle in each of the halos will be denoted by $r$ with the index $h$, like halo, for the more massive of the two merging halos and index $s$, like satellite, for the less massive halo. Throughout this paper we will use these indexes to refer quantities to the more and less massive of the merging halos.

In the following we will assume that each of the two point masses moves with the average velocity of the particles inside the virial radius of the corresponding halo.

Knowing the average velocity and mass inside the virial radii of the merging halos it is possible to solve the gravitational two body problem analytically by making the transition to the equivalent one-body problem of the reduced mass system (e.g. Goldstein et al. 2002). The equation of motion reads

$\mu \ddot{\mathbf{r}}=-G \frac{M_{h} M_{s}}{r^{2}} \frac{\boldsymbol{r}}{r}$

with $M_{h}$ and $M_{s}$ being the mass inside the virial radius of the more and less massive of the merging halos, respectively. The reduced mass is defined by

$\mu \equiv \frac{M_{h} M_{s}}{M_{h}+M_{s}}$

Equation (1) describes the motion of a fictitious particle of mass $\mu$ moving around a point at a distance $r=\left|r_{h}-r_{s}\right|$. To specify the orbit we calculate the total energy and orbital angular momentum according to

$E=\frac{1}{2} \mu \dot{r}^{2}-\frac{G M_{h} M_{s}}{r}$

and

$L=\mu \boldsymbol{r} \times \dot{\boldsymbol{r}}$,

respectively. In this study we also investigate the pericenter distance $r_{\text {peri }}$ and the eccentricity $e$ of orbits, which we can readily calculate from the energy and orbital angular momentum by

$e=\sqrt{1+\frac{2 E L^{2}}{\mu\left(G M_{h} M_{s}\right)^{2}}}$

and

$r_{\text {peri }}=\frac{L^{2}}{(1+e) \mu G M_{h} M_{s}}$.

In the following we will identify orbits according to their total energy and eccentricity into hyperbolic $(E>0, e>1)$, parabolic $(E=0, e=1)$ and elliptic $(E<0, e<1)$ orbits.

\subsection{Limits on the two-body approximation}

As summarised in the previous section we assume the two merging halos to be fairly isolated and without experiencing any other severe interactions before they merge. This however, is not guaranteed and needs to be checked. We calculated the total energy and the orbital angular momentum of the orbits of the two halos at two simulation outputs before they merge. To do so we followed the most massive progenitors backward in time and analysed their orbital parameters, including all halos with more than 50 particles and with a mass ratio of $4: 1$ or less. The filled circles in top left panel of Fig. 1 show the total energy 

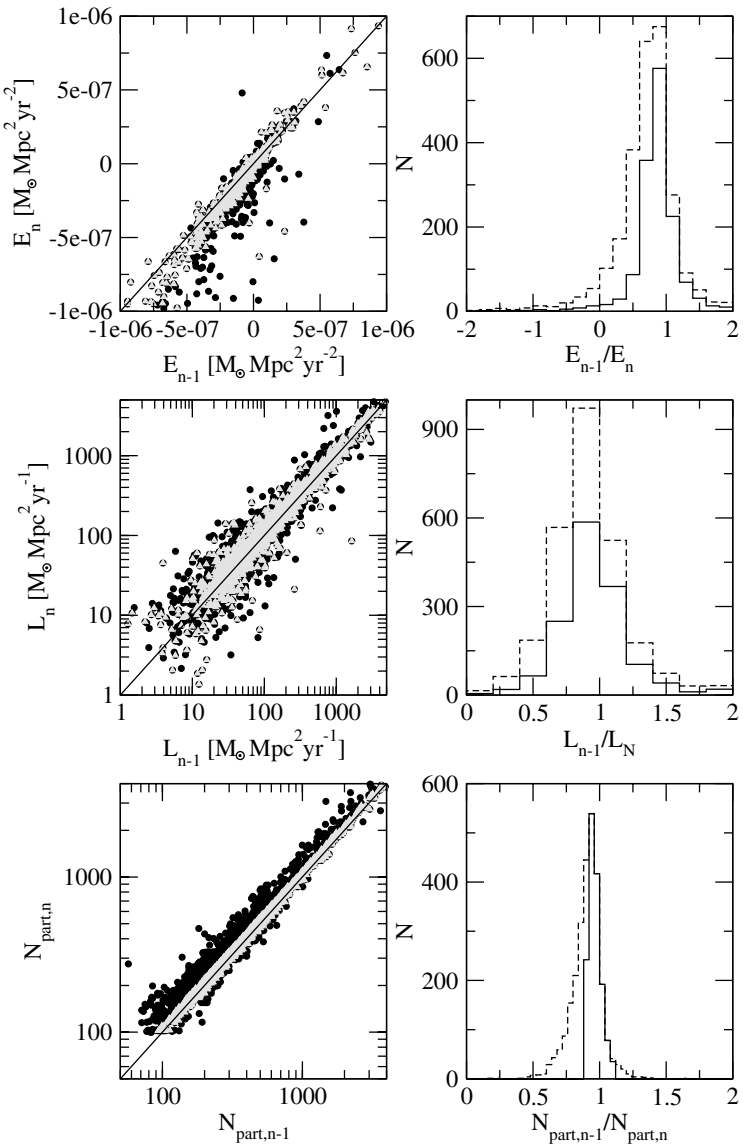

Fig. 1. Upper left panel: total energy of the two halo system. Upper right panel: distribution of ratios $E_{n-1} / E_{n}$. Middle panel: orbital angular momentum of the two halo system. Middle right panel: distribution of ratios $L_{n-1} / L_{n}$. Lower panel: mass of the progenitors in units of particle mass in the simulation. Lower right panel: distribution of ratios $N_{\text {part }, n-1} / N_{\text {part }, n}$. The indexes $n$ and $n-1$ refer to one and two simulation time steps before the merger. The circles show all identified mergers, and the triangles only those when applying the mass criterion. Dashed histograms show the distribution for all mergers and solid histograms only for those satisfying the mass criterion.

of the merging halos $E_{n}$ and $E_{n-1}$, one and two time steps before the merger, respectively. As can be seen the energies seem to be not too different between the two time steps, with a tendency for bound orbits at time step $n-1$ to become more bound at $n$. The dashed histogram in top right panel shows the distribution of ratios $E_{n-1} / E_{n}$ for all identified mergers. The distribution is skewed toward values smaller than one which confirms the tendency of the orbits to become more bound between two time steps. The filled circles in the left middle panel of the same figure shows the correlation between the orbital angular momentum at two preceding time steps before the merger. The dashed histogram in the right middle panel shows the distribution of the ratio of these orbital angular momenta. In general the orbital angular momentum between two outputs does not change. However we find that there is a considerable scatter which is symmetrically distributed around $L_{n}=L_{n-1}$.

The aim of this work is to present initial conditions for high resolution simulations of isolated merging galaxies. This means that we ideally would like the energy and angular momentum to be conserved or not to change significantly while the two merging halos are well separated on their approach. As can be seen in the lower left panel of Fig. 1 many of the progenitors are not isolated in the sense that they do not accrete further mass before they merge. To choose fairly isolated halos we therefore decide to only consider those mergers in which $1.11\left(M_{h, n}+M_{s, n}\right) \geq M_{h, n-1}+M_{s, n-1} \geq 0.9\left(M_{h, n}+M_{s, n}\right)$. The result of this selection criterion is shown by the grey triangles in the left panels of Fig. 1. In all cases the correlations between the quantities at the different redshifts become tighter. In addition we find that the distribution in the ratio of energies is now centred around a value of one (solid histogram right top panel). The shape of the distribution in the ratios of the angular momenta does not change much though it becomes a bit narrower. In the following we will use above mass criterion to select mergers for the sample used here. This criteria roughly rejects $50 \%$ of the mergers detected in the simulation.

\section{Orbital parameters $r_{\text {peri }}$ and $e$ for major mergers}

We start by analysing the dependencies and correlations of the pericenter distance $r_{\text {peri }}$ and the eccentricity $e$ of merging halos extracted from the simulation data at one output prior to the merger. We restrict ourselves to mergers with mass ratios of 4:1 or less as numerical models have shown that larger mass ratios do not resemble elliptical galaxies anymore as the more massive disk is not destroyed (Barnes \& Hernquist 1992; Naab \& Burkert 2003).

In Fig. 2 the distribution of eccentricities $e$ of merging halos as a function of the minimum mass of the progenitor halos is shown. Roughly half of the orbits are parabolic or very close to parabolic. We find $\sim 40 \%$ of the orbits in the range $e=1 \pm 0.1$. This result is independent of the minimum mass cut applied. $N$-body simulations of merging galaxies assume parabolic orbits (e.g. Barnes 1988), which is only the case for half of the mergers we find. In Fig. 3 we show the dependence of our results on the mass ratio of the major mergers. We find no dependence with the mass ratio, which indicates a self-similarity of the formation process. As many characteristic properties of elliptical galaxies as e.g. the amount of rotation are determined by the mass ratio (Naab \& Burkert 2003) the cosmological models would predict that massive and low-mass ellipticals should have similar properties which is not in agreement with observations. In contrast with the theoretical predictions luminous ellipticals are preferentially anisotropic and slowly rotating while less luminous ellipticals rotate fast and appear isotropic (Bender 1988). The distribution of the pericenter distance in units of the virial radius of the more massive progenitor halo and its dependence on minimum mass is shown in the left panels of Fig. 4. The distribution shows no significant dependence on the minimum mass. Small pericenter are more frequent than larger ones. This is actually what one would expect, because halos which are on orbits leading to a very close encounter are more likely to merge than those which pass each other from very far. Roughly $85 \%$ of the mergers have pericenter distances which are larger than $0.1 r_{\text {vir }, h}$. Figure 4 show that the dependence on the mass ratio of the mergers is negligibly small. Merger simulations usually adopt initial 

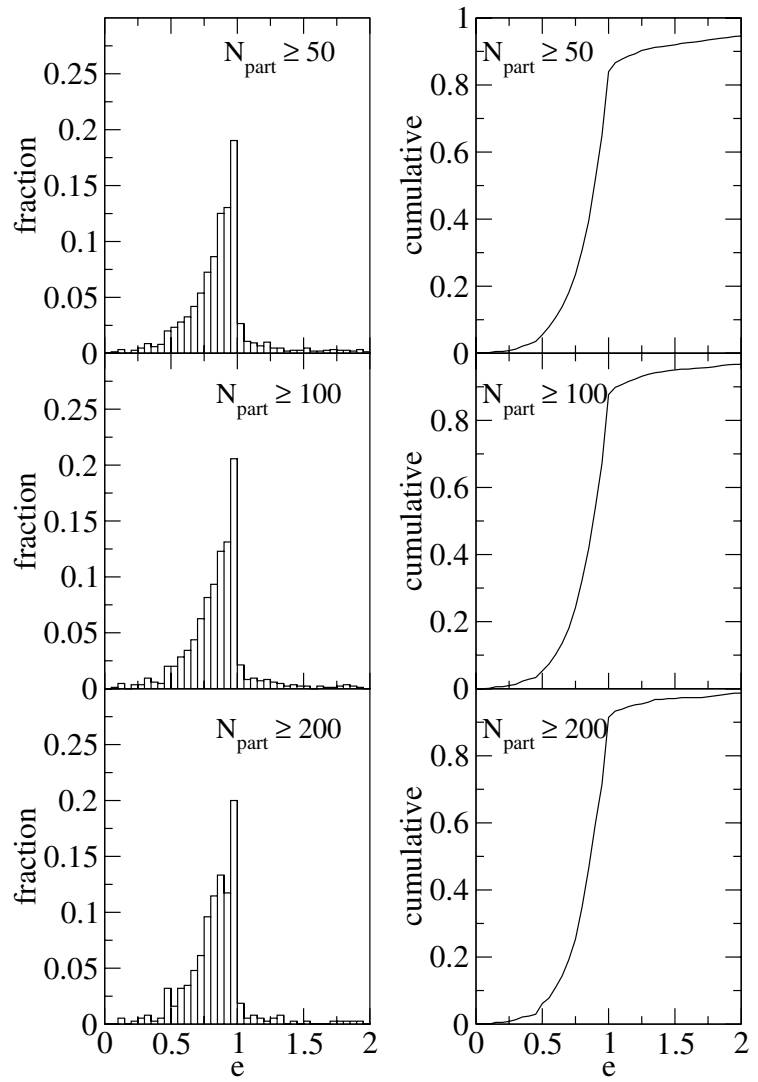

Fig. 2. Left panel: eccentricity distribution of merging halos with mass larger than $N_{\text {part }} \cdot 2 \times 10^{10} M_{\odot} . N_{\text {part }}$ is the number of dark matter particles. Right panel: corresponding cumulative fraction of eccentricities.

conditions with small pericenter distances which are typically of order $0.05 r_{\mathrm{vir}, h}$, leading to fast merging that saves computational time. This assumption is not in agreement with cosmological models and might affect the inferred structure of merger remnants using numerical simulations. According to the reduced two-body problem $r_{\text {peri }} \propto L^{2}$, which means that the orbital angular momentum in typical merger simulations is less than that for merging halos in self-consistent cosmological simulations. Less angular momentum will therefore be transferred during the merger process and the structure of the remnant could be different. In Fig. 5 we correlate the pericenter distance in units of $\mathrm{Mpc}$ to the pericenter distance in units of $r_{\mathrm{vir}, h}$. We find that on average roughly $50 \%$ of all mergers have pericenter distances less than $0.5 r_{\mathrm{vir}, h}$. About $70 \%$ of all mergers lead to a first pericenter passage which penetrates the virial radius of the larger halo $r_{\mathrm{vir}, h}$.

Since we have found no dependence on the minimum mass and on the mass ratio we continue our investigations using as a standard assumption $M_{\min }=10^{12} M_{\odot}$ which corresponds to the typical halo size of massive galaxies and $M_{h} / M_{s} \leq 4$. Not every random orbit is going to lead to a merger and it is important to see if a preferred orbit configuration exists leading to mergers. In Fig. 6 we correlate $r_{\text {peri }}$ and $e$. Mergers with $r_{\text {peri }} \leq 0.1 r_{\text {vir }, h}$ are almost all on parabolic orbits with $e \sim 1$. Orbits with $r_{\text {peri }}>0.1 r_{\text {vir }, h}$ have a scatter in $e$ which increases with pericenter distance toward bound orbits. The same
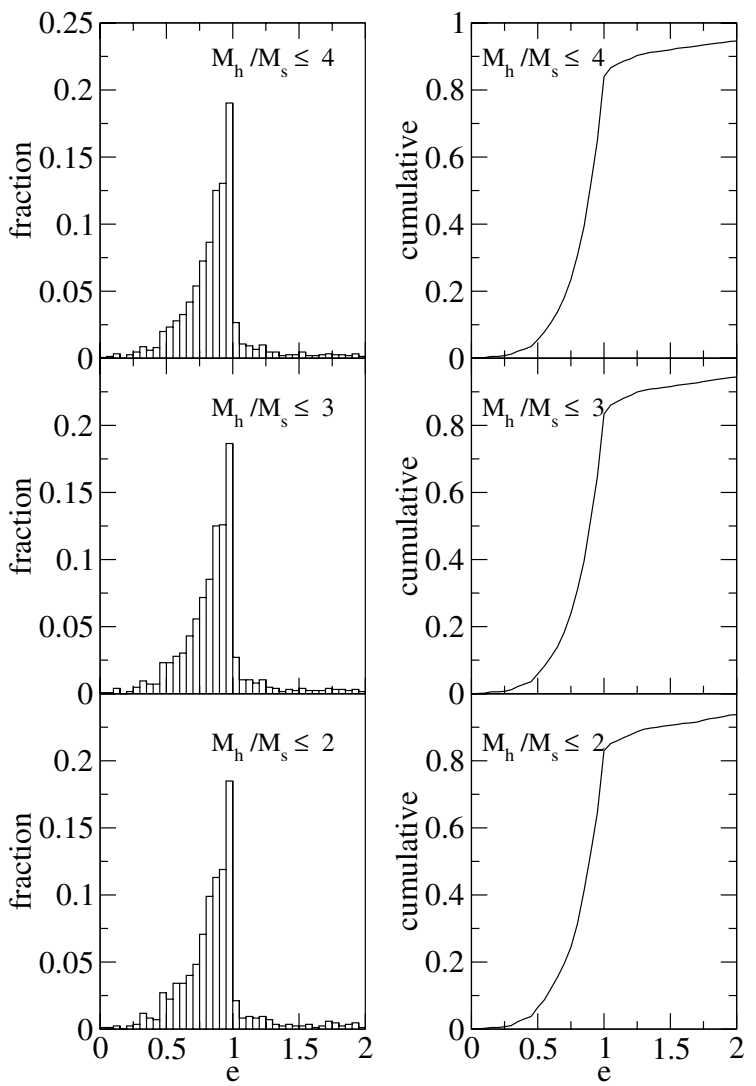

Fig. 3. Left panel: fraction of merging halos with mass larger than $10^{12} M_{\odot}$ and different mass rations $M_{h} / M_{s}$, on initial orbits with eccentricity $e$. Right panel: corresponding cumulative fraction of eccentricities.

behaviour is found looking at the correlation between eccentricities and pericenter distances in units of Mpc.

The expectation from the two-body solution is that the specific angular momentum $L_{\mathrm{sp}} \equiv L / \mu$ for remnants of the same mass $M_{h}+M_{s}$ is proportional to $r_{\text {peri }}^{1 / 2}$ with a scatter because of different eccentricities of the orbits. In Fig. 7 we show the correlations found between these two quantities. The lines in the left and right panel of Fig. 7 are power law fits to the data with

$L_{\mathrm{sp}}=1.11 \times 10^{-10}\left(\frac{r_{\text {peri }}}{r_{\mathrm{vir}, h}}\right)^{0.48} \frac{\mathrm{Mpc}^{2}}{\mathrm{yr}}$

for the pericenter distance in units of $r_{\mathrm{vir}, h}$ and

$L_{\mathrm{sp}}=2.84 \times 10^{-10}\left(\frac{r_{\mathrm{peri}}}{\mathrm{Mpc}}\right)^{0.53} \frac{\mathrm{Mpc}^{2}}{\mathrm{yr}}$

for the pericenter in units of Mpc. The fits show that the data is following the trend of $L_{\mathrm{sp}} \propto r_{\text {peri }}^{1 / 2}$. The larger scatter in the correlation with $r_{\text {peri }}$ in units of $r_{\mathrm{vir}, h}$ is due to additional scatter introduced on the horizontal axis by the variation in the values of $r_{\mathrm{vir}, h}$ at a given $r_{\text {peri }}$.

Hyperbolic encounters are often characterised by the impact parameter $b$. The impact parameter is defined by the spatial separation perpendicular to the initial velocity $V(t=-\infty)$ which has the value

$V_{-\infty}=\sqrt{\frac{2 E}{\mu}}$. 


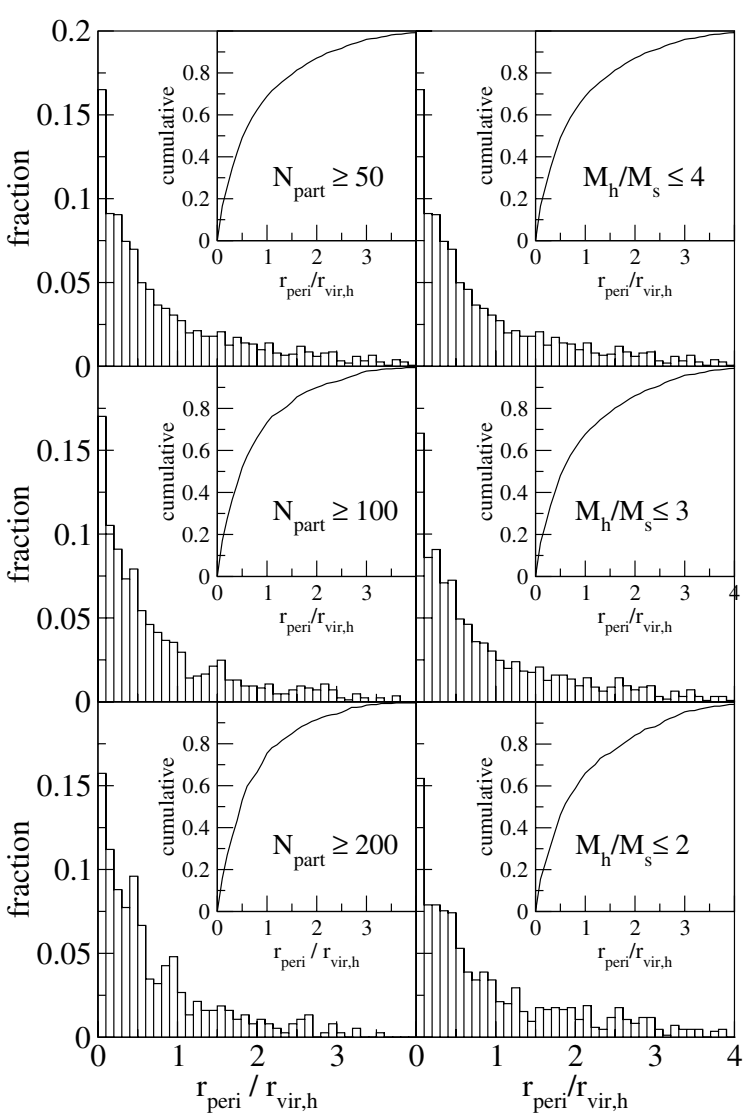

Fig. 4. Left panel: fraction of merging halos with different pericenter distances in units of the more massive progenitor's virial radius $r_{\mathrm{vir}, h}$ and with progenitor masses larger than $N_{\text {part }} \cdot 2 \times 10^{10} M_{\odot}$. The corresponding cumulative fraction of pericenter distances are also shown. All results are shown for mergers with a mass ratio of $M_{h} / M_{s} \leq 4$. Right panel: fraction of merging halos with mass larger than $10^{12} M_{\odot}$ and different mass ratios $M_{h} / M_{s}$, on orbits with different pericenter distances in units of the more massive progenitor's virial radius $r_{\mathrm{vir}, h}$. Cumulative fractions are shown too.

Because of the conservation of orbital angular momentum the impact parameter is given by

$b=\frac{L}{\mu V_{-\infty}}=\frac{L}{\mu \sqrt{2 E / \mu}}$.

Figure 8 shows the probability distribution function of impact parameters which shows a peak around $b \approx 0.5$. We fit the distribution with a function of the form:

$\frac{\mathrm{d} P}{\mathrm{~d} x} \mathrm{~d} x=\frac{1}{a_{2}} \frac{a_{0}}{a_{1}}\left(\frac{x}{a_{1}}\right)^{a_{0}-1} \exp \left(-\frac{x}{a_{1}}\right)^{a_{0}} \mathrm{~d} x$,

and we present the best fit parameters in Table 1. In the upper graph of Fig. 9 we show the correlation between the impact parameter and the pericenter distance. The line is a power-law fit to the data with

$r_{\text {peri }}=0.3 b^{0.72}$.

The data shows a large scatter which makes the power-law fit a rather poor fit. However, to guide the eye and show the general trend we include this fit. The lower graph of Fig. 9 displays the correlation between eccentricity and impact parameter of the

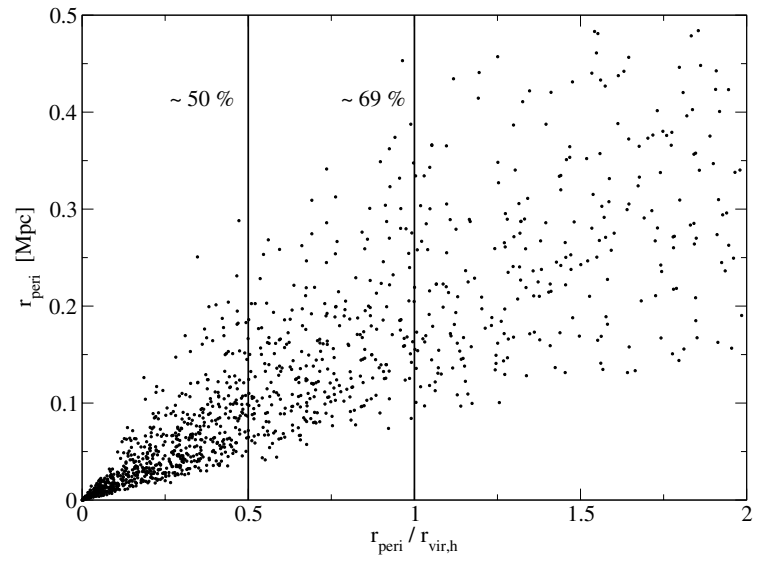

Fig. 5. Pericenter distance in units of Mpc against pericenter distance in units of $r_{\mathrm{vir}, h}$. The values at each line indicate the completeness limit, meaning the fraction of mergers with $r_{\text {peri }} / r_{\mathrm{vir}, h}<0.5$ and 1 . Results are shown for progenitor halos of mass larger than $10^{12} M_{\odot}$ and mergers with mass ratio $M_{h} / M_{s} \leq 4$.

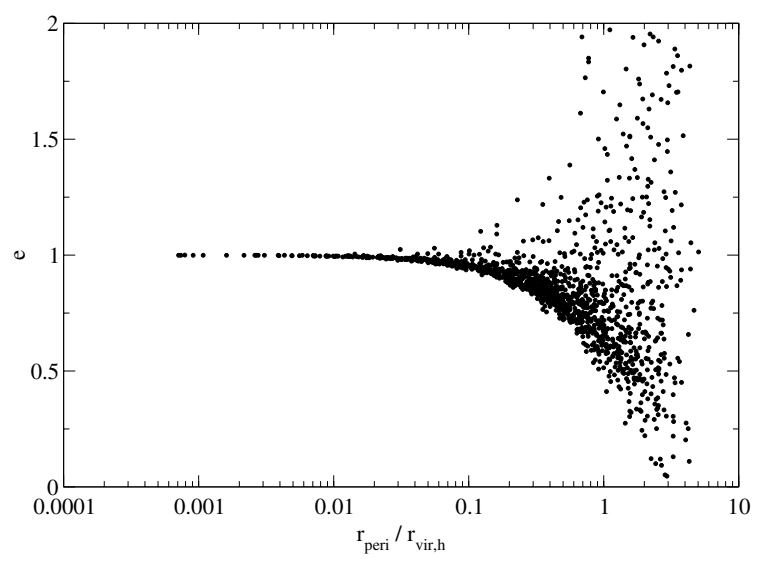

Fig. 6. Correlation between the eccentricity and pericenter distance of merging halos found in the simulation. Results are shown for progenitor halos of mass larger than $10^{12} M_{\odot}$ and mergers with mass ratio $M_{h} / M_{s} \leq 4$.

encounter. Again it becomes evident that many of the orbits are close to be parabolic and that only a small fraction is significant different from parabolic. Encounters having $e>1$ merge very slowly if at all. This explains why only hyperbolic encounters with small impact parameters $b<1 \mathrm{Mpc}$, corresponding to a close flyby, lead to mergers.

Another parameter commonly used to describe bound orbits with $E<0$ is the circularity $\epsilon$ defined as the ratio of the orbital angular momentum to the angular momentum of a circular orbit with the same energy. The circularity of a bound orbit can be derived applying the virial theorem $U=-2 T$. With

$r_{\mathrm{circ}}=\frac{G M_{h} M_{s}}{2 E}, \quad V_{\mathrm{circ}}=\sqrt{\frac{-2 E}{\mu}}$

and the angular momentum

$L_{\text {circ }}=r_{\text {circ }} \mu V_{\text {circ }}$, 


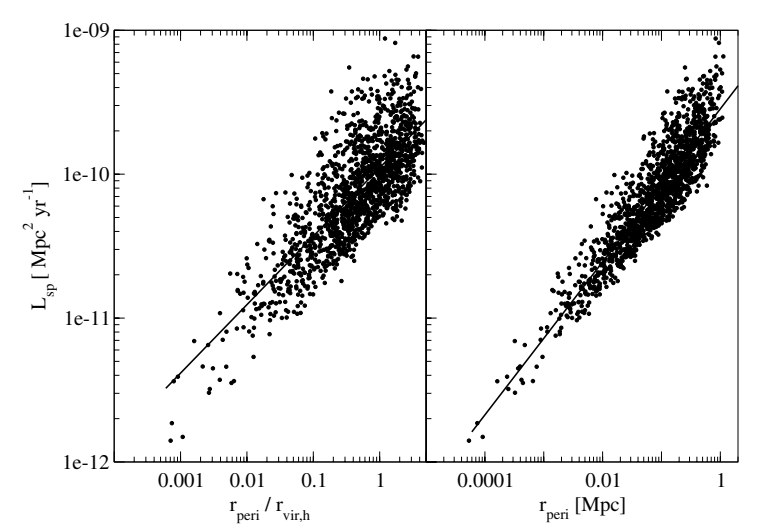

Fig. 7. Correlation between the specific angular momentum $L_{\mathrm{sp}}$ of the orbit and its pericenter distance in units of $r_{\mathrm{vir}, h}$ (left panel) and Mpc (right panel). Lines represent power law fits to the data. Results are shown for progenitor halos of mass larger than $10^{12} M_{\odot}$ and mergers with mass ratio $M_{h} / M_{s} \leq 4$.

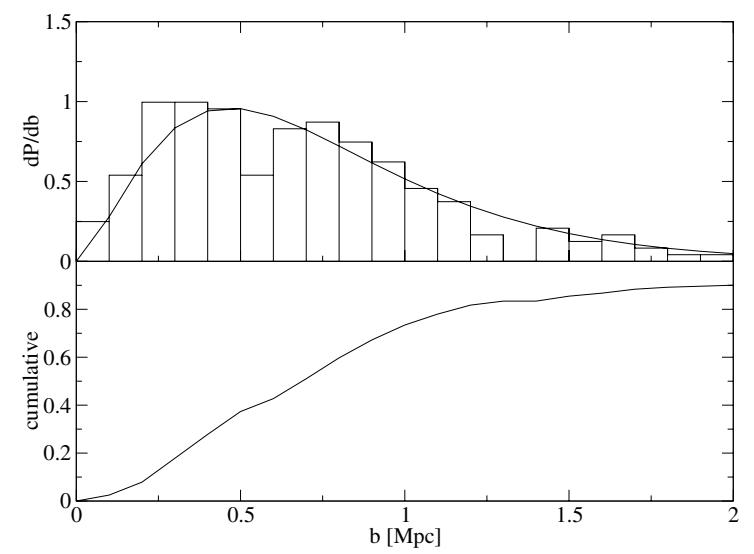

Fig. 8. Upper graph: probability density of hyperbolic orbits with different impact parameter $b$. Lower graph: to the upper graph corresponding cumulative fraction. Results are shown for progenitor halos of mass larger than $10^{12} M_{\odot}$ and mergers with mass ratio $M_{h} / M_{s} \leq 4$.

the circularity becomes

$\epsilon=\frac{L}{L_{\text {circ }}}=\frac{L}{r_{\text {circ }} \mu V_{\text {circ }}}$.

From Eq. (13) one sees that the circularity can only be defined for orbits with $E<0$. Manipulating the appropriate equations gives the following relation for the circularity and eccentricity of an orbit:

$\epsilon=\sqrt{1-e^{2}}$.

The upper graph of Fig. 10 presents the distribution of circularities found. This result agrees with that of Tormen (1997) who found the circularities to be distributed with a peak around $\epsilon \approx 0.5$. However, in their analyses they considered only minor mergers $M_{h} / M_{s} \gg 4$ in a cluster environment, where the enhanced gravitational field might lead to a change of the circularity distribution. Our results show that these effects seem to play not an important role, and that the circularity distribution seems to be universal. An important consequence for semianalytic modelling is that the circularity $\epsilon$ used to calculate the dynamical friction time scale for major mergers must not be
Table 1. Best-fit parameters using Eq. (11) to the distributions shown in Figs. 8, 19 and 20.

\begin{tabular}{cccc}
\hline \hline$x$ & $a_{0}$ & $a_{1}$ & $a_{2}$ \\
\hline$b$ & 2.636 & 0.753 & 0.326 \\
$L / S_{h}$ & 1.727 & 7.408 & 0.662 \\
$L / S_{s}$ & 1.624 & 14.132 & 0.726 \\
$f$ & 2.638 & 1.2 & 0.307 \\
\hline
\end{tabular}

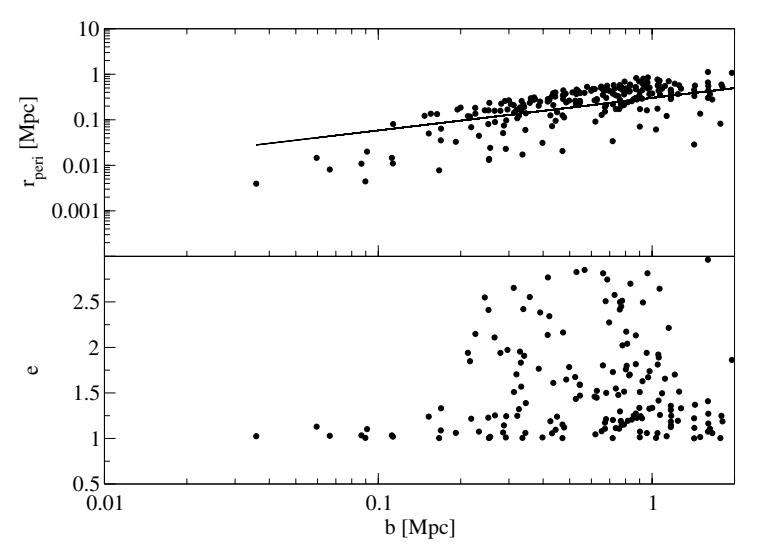

Fig. 9. Upper graph: correlation between pericenter distance and impact parameter of hyperbolic orbits leading to mergers. Lower graph: correlation between the eccentricity and impact parameter corresponding to the orbits in the upper graph. Results are shown for progenitor halos of mass larger than $10^{12} M_{\odot}$ and mergers with mass ratio $M_{h} / M_{s} \leq 4$.

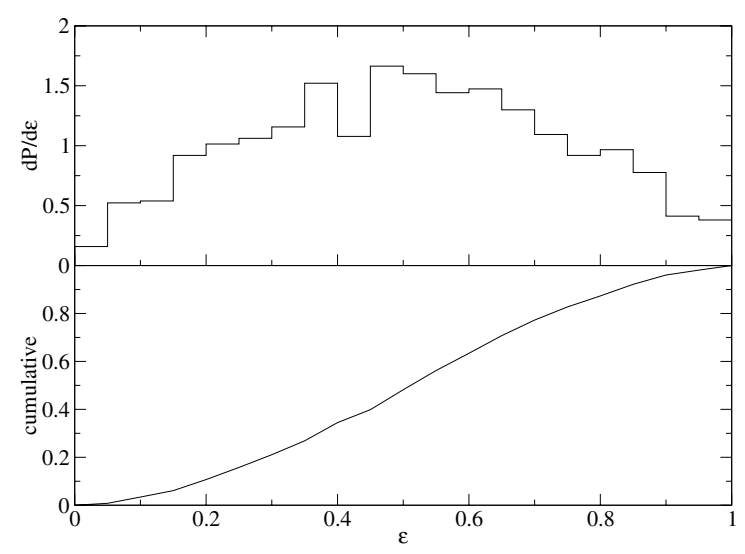

Fig. 10. Upper graph: probability density of bound orbits with different circularity. Lower graph: to the upper graph corresponding cumulative fraction. Results are shown for progenitor halos of mass larger than $10^{12} M_{\odot}$ and mergers with mass ratio $M_{h} / M_{s} \leq 4$.

drawn from a uniform distribution as e.g. done in Kauffmann et al. (1999) but from the distribution found here.

Figure 11 shows that the correlation between circularity and the pericenter distance. We find that the data can be fitted well by following power-law

$r_{\text {peri }}=0.38 \epsilon^{2.17} \mathrm{Mpc}$. 


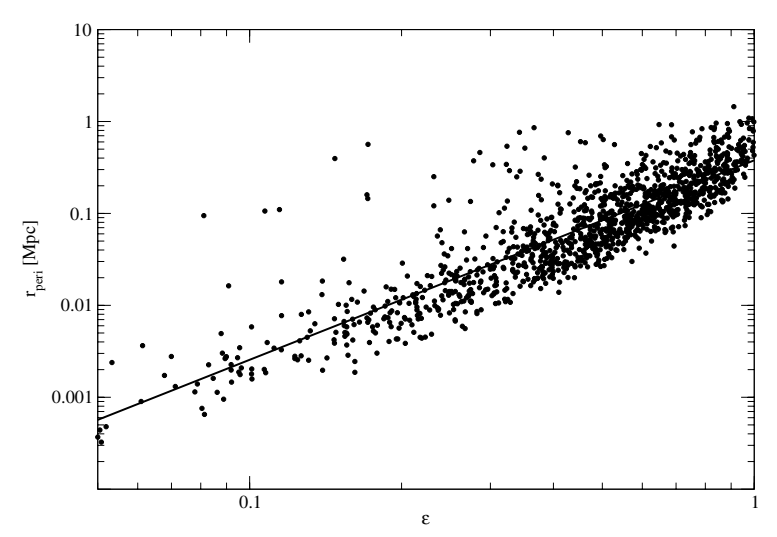

Fig. 11. Correlation between pericenter distance and circularity of bound orbits leading to mergers. Results are shown for progenitor halos of mass larger than $10^{12} M_{\odot}$ and mergers with mass ration $M_{h} / M_{s} \leq 4$.

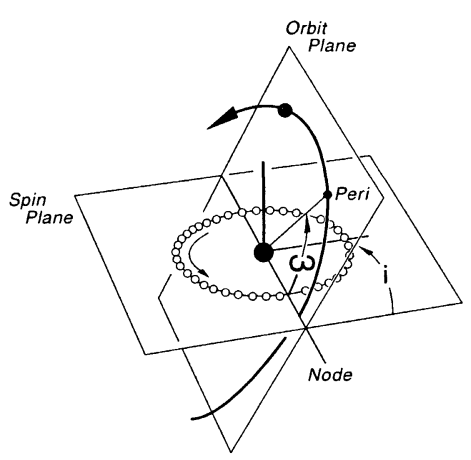

Fig. 12. Definition of the angles $i$ and $\omega$ following TT72 (figure reproduced with permission of A. Toomre).

\section{Parameters $\omega$ and $i$}

If halos have spin, the orbital parameters discussed in the last section are not enough to fully describe the geometry of the encounter. Additional constraints on the position of the spin vectors $\boldsymbol{S}$ of both halos are required. These spin vectors are in general expected to align with the spin vectors of the galactic disks that form by gas infall and kinetic energy dissipation. In calculating the spin vectors we follow Lemson \& Kauffmann (1999) who derived spin parameters from a similar GIF simulation. They found that for halos with more than 70 particles halo properties are calculated reliably. Additionally they require that between 35 and 95 per cent of the friends-offriends mass of the halo are inside of the virial radius. They find that 80 per cent of the halos satisfy these requirements. In our analysis we adopt limits of 50 particles or more per halo and between 50 and 95 per cent of the friends-of-friends mass inside the virial radius. In that way we make sure that we get results which are not influenced by ambiguities in the way we treat the particles inside each halo. In Fig. 12 the definition of the two corresponding angles is shown. The angle $i$ is defined in the rest frame of the halo as the angle between the spin plane of the halo and the orbital plane of the satellite; and in the rest frame of the satellite as the angle between the spin plane

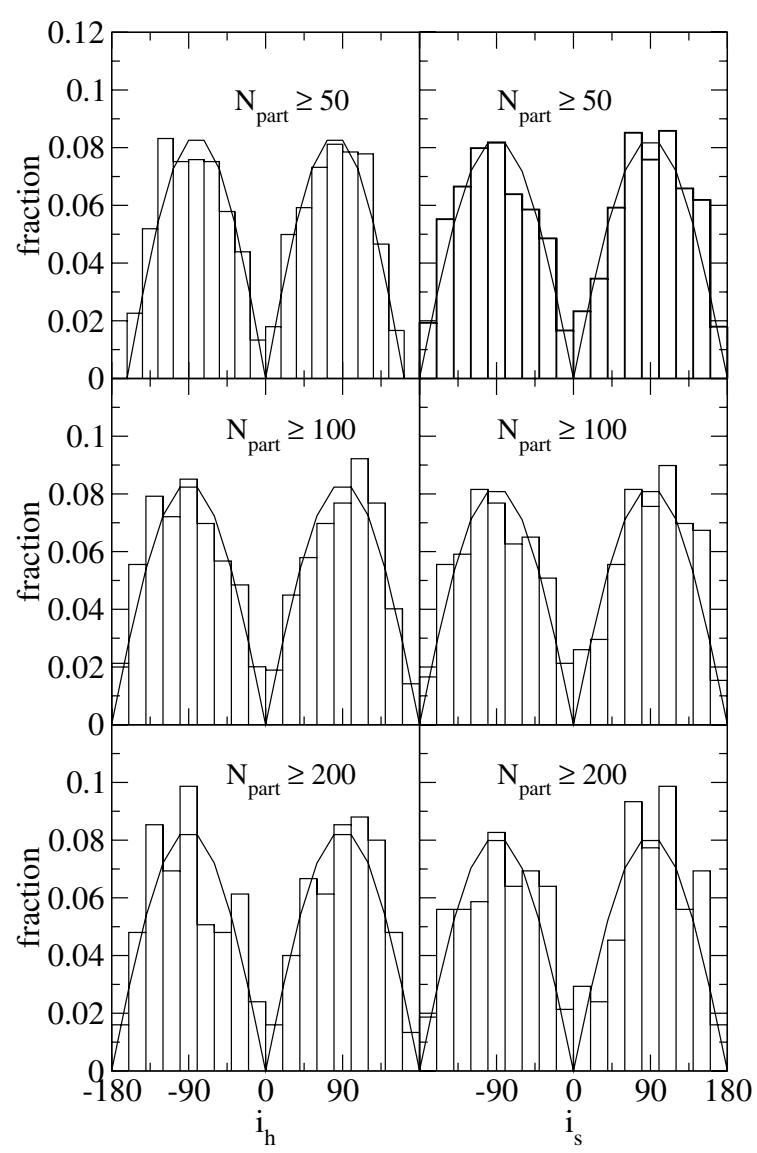

Fig. 13. Left column: angle between halo spin plan and orbital plane for different choices of minimum progenitor mass and a major merger definition of $M_{h} / M_{s} \leq 4$. Right column: same as left column but now for the angle between the spin plane of the satellite and orbital plane.

of the satellite and the orbital plane of the halo. By spin and orbital plane we refer to the planes perpendicular to the spin and orbital angular momentum vector, respectively. These two angles $i_{h}$ and $i_{s}$ are independent and by definition $|i| \leq 180^{\circ}$, where $i=0^{\circ}$ is a prograde and $i=180^{\circ}$ a retrograde encounter. Additionally, the pericentric argument $\omega$, is defined as the angle between the line of nodes and separation vector at pericenter, and has values ranging from $\omega=-90^{\circ}$ to $\omega=90^{\circ}$. It is not defined for $i=0^{\circ}$ or $i=180^{\circ}$.

Figures 13-15 show the correlation between the angles $i$ and $\omega$ and the minimum mass of progenitor halos or the mass ratios of the mergers. The angles $i_{h}$ and $i_{s}$ are distributed following a sinus function. The fit gets naturally poorer at high minimum masses because of the smaller sample of halos. The solid lines in Figs. 13 and 14 are fits of the form $\propto|\sin (x)|$. If the angle between two vectors is sinus-distributed, the two vectors have no correlation. This can be understood from looking at the probability of drawing a random vector pointing from the centre of a sphere to its surface. If every point on the surface is equally likely, the probability of finding an angle $i$ for example between the $\mathrm{x}$-axis and a random vector will be proportional to $\sin (i)$. We therefore conclude that the spin plane and the orbital angular momentum plane have no correlation. Inspection of Fig. 15 reveals that the angle $\omega$ is equally distributed and independent of the minimum halo mass and major merger 


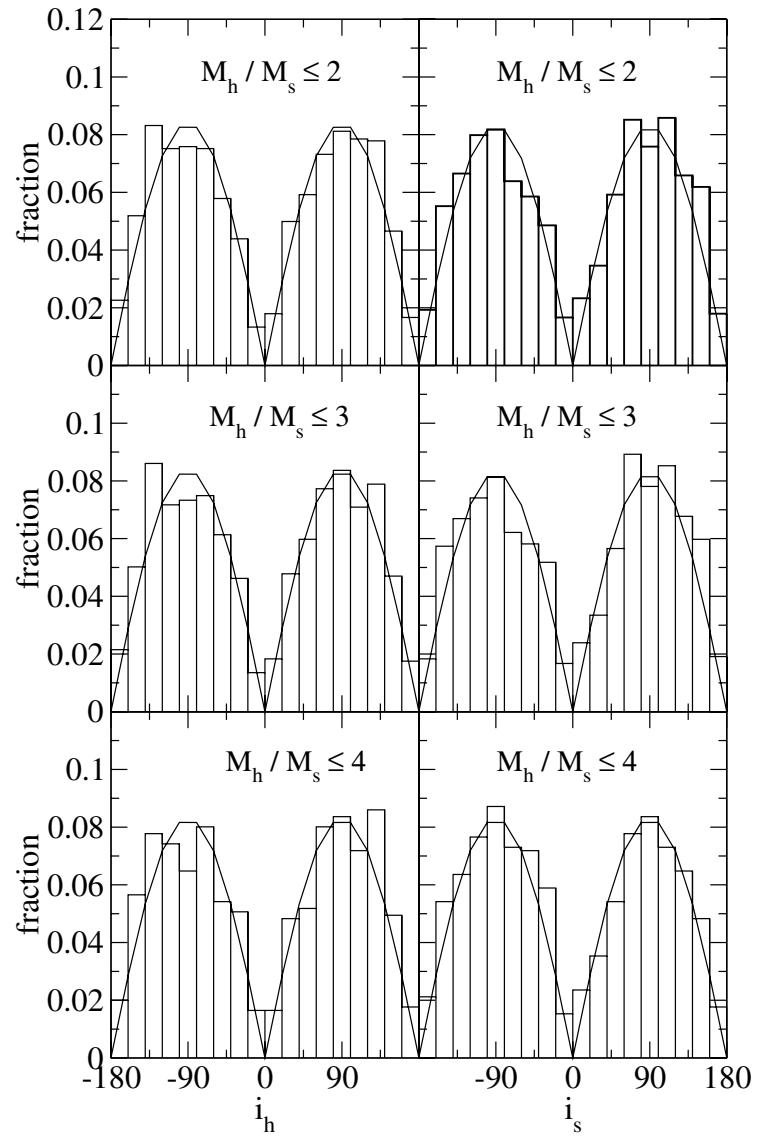

Fig. 14. Left column: angle between halo spin plan and orbital plane for different choices of major merger definition and fixed minimum progenitor mass of $10^{12} M_{\odot}$. Right column: same as left column but now for the angle between the spin plane of the satellite and orbital plane.

definition. In contrast to $i, \omega$ is defined by the position of a vector lying in a predefined plane. As a result an equal distribution in $\omega$ means that this angle is randomly distributed.

Defining $\kappa$ as the angle between the spin planes, one would expect from the results presented above that the spin vectors are also not correlated with each other, and that $\kappa$ should be sinus-like distributed. The distribution of $\kappa$ is shown in Fig. 16 and is indeed sinus like.

It is important to investigate possible correlations between the orbital parameters $r_{\text {peri }}$ and $e$ and the angles introduced above. Figure 17 illustrates the correlation between the angle $i_{s}$ and the orbital parameters. The orbital parameters are not correlated with $i_{s}$. The same results are found for $i_{h}$. The pericentric argument $\omega_{s}$ shows no correlation with the orbital parameters as shown in Fig. 18. The results are the same for $\omega_{h}$.

Semi-analytic models (Maller et al. 2002; Vitvitska et al. 2002) assume that during mergers the orbital angular momentum gets transformed into spin of the remnant halo. These models and models in which angular momentum is acquired by tidal torques e.g. Porciani et al. (2002) reproduce the spin distribution of halos found in $N$-body simulations. However the merger picture for the build up of halo spins uses some assumptions which still need confirmation by $N$-body simulations. As a first step the amount of angular momentum in the orbit must be

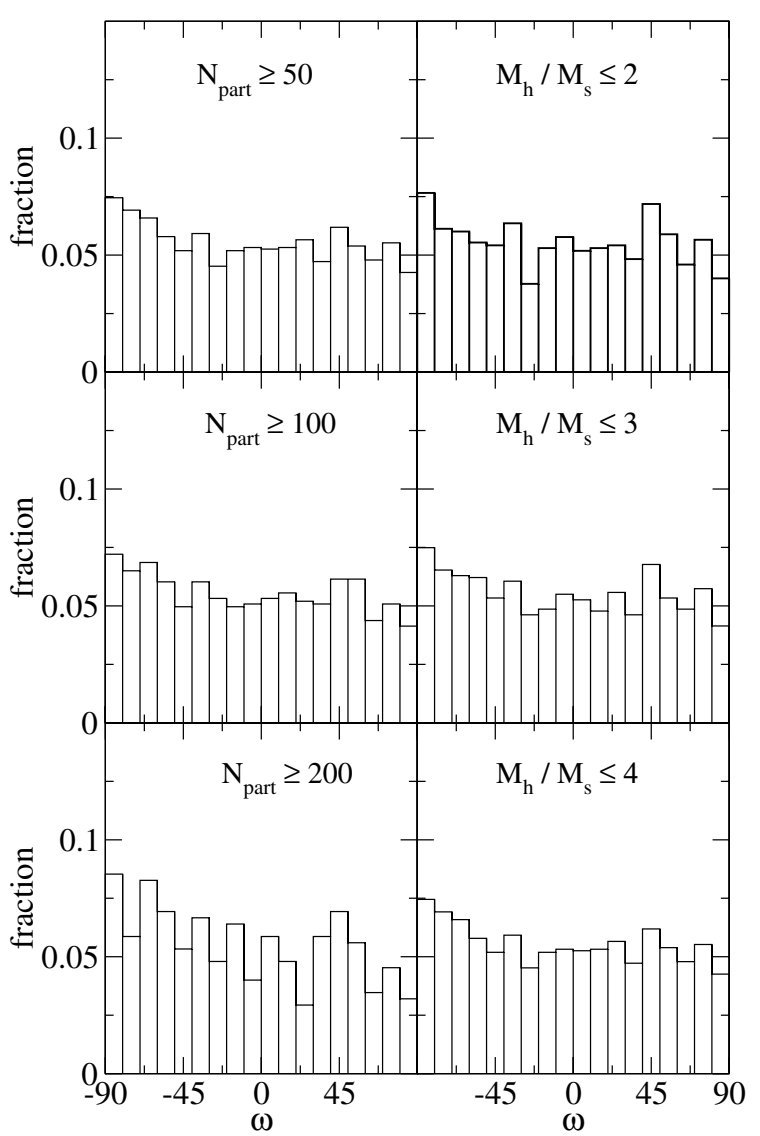

Fig. 15. Left column: angle between pericenter vector and node line for different choices of minimum progenitor mass and a major merger definition of $M_{h} / M_{s} \leq 4$. Right column: same as left column but now for fixed minimum progenitor mass of $10^{12} M_{\odot}$ and different choices of major merger definition. Results are the same for $\omega_{s}$ and $\omega_{h}$.

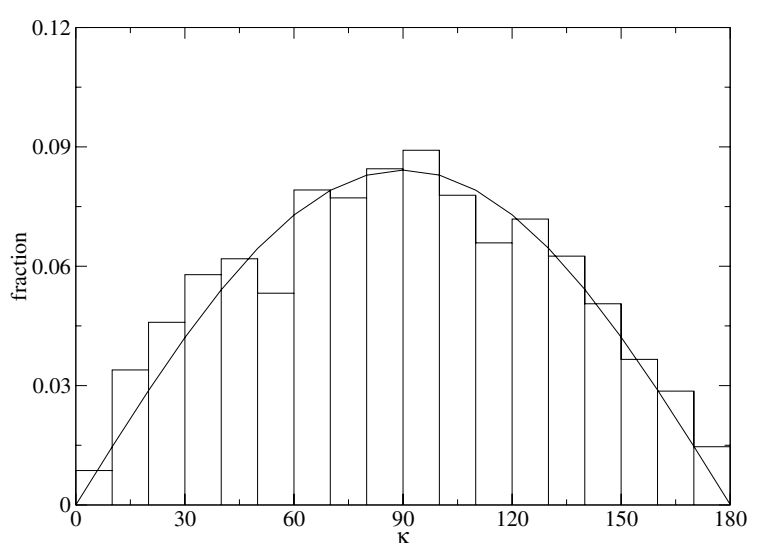

Fig. 16. The distribution of angles $\kappa$ between the two spin planes. Results are shown for progenitor halos of mass larger than $10^{12} M_{\odot}$ and mergers with mass ratio $M_{h} / M_{s} \leq 4$.

investigated. Figure 19 shows the distribution of the fraction of orbital angular momentum to spin of the halos $S_{h}$ and spin of the satellite $S_{s}$.

Maller et al. (2002) define a parameter $f$ for mergers

$f=\frac{L}{V_{\mathrm{vir}, h} r_{\mathrm{vir}, h} \mu}$ 


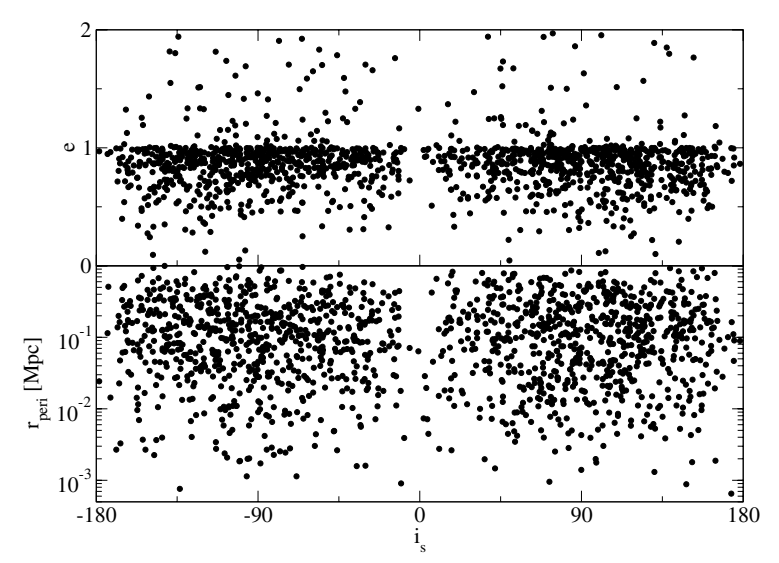

Fig. 17. Upper graph: correlation between $i_{s}$ of the satellite and the eccentricities of orbits leading to mergers. Lower graph: correlation between the $r_{\text {peri }}$ and $i_{s}$ corresponding to the orbits in the upper graph. Results are shown for progenitor halos of mass larger than $10^{12} M_{\odot}$ and mergers with mass ratio $M_{h} / M_{s} \leq 4$.

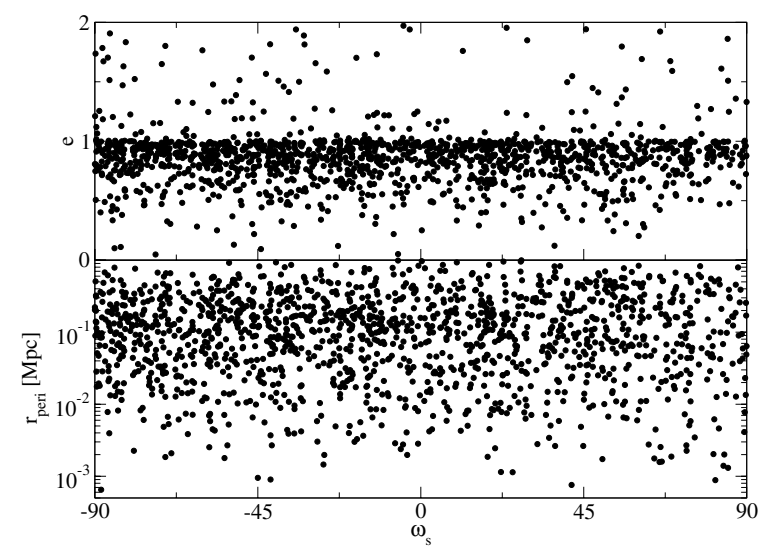

Fig. 18. Upper graph: correlation between $\omega_{s}$ and $e$ for orbits leading to mergers. Lower graph: correlation between $r_{\text {peri }}$ and $\omega_{s}$, corresponding to the orbits in the upper graph. Results are shown for progenitor halos of mass larger than $10^{12} M_{\odot}$ and mergers with mass ratio $M_{h} / M_{s} \leq 4$.

with $V_{\mathrm{vir}, h}$ as the circular velocity of the more massive progenitor. The value of this parameter is set to be $f \sim 0.42$ for their model, in which spin is acquired from orbital angular momentum. In Fig. 20 the distribution of $f$ is displayed. We fit the distributions in Figs. 19 and 20 with functions of the form presented in Eq. (11), and the fitting parameters can be found in Table 1 . The distribution for $f$ peaks at $\sim 0.6$ and has its mean at $\sim 1$. which disagrees with the value required in the model of Maller et al. (2002).

\section{Discussion and conclusions}

We have investigated the orbital parameters of merging dark matter halos in a cosmological large scale simulation finding that the parameters used generally in simulations of merging galaxies are in agreement with those found in the large scale simulation. However, parameters used in merger simulations up to now only occupy a very small part of the region of parameter space covered by the results from the large scale simulation.

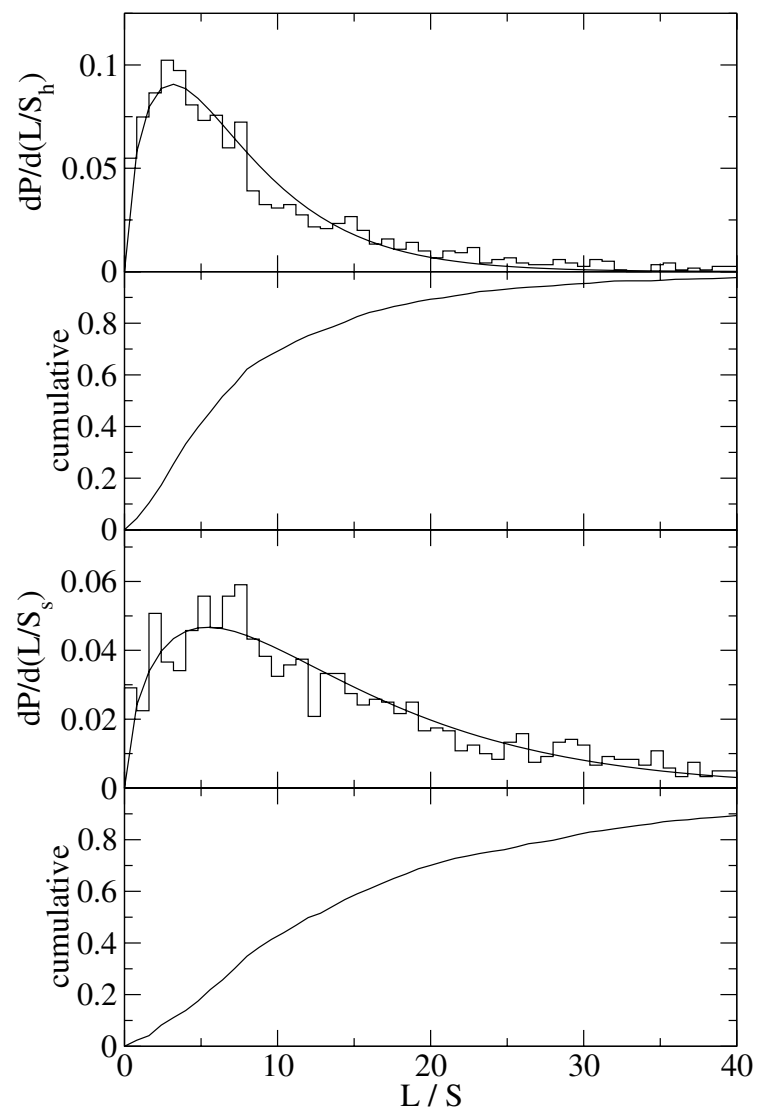

Fig. 19. The upper two graphs show the distribution and corresponding cumulative fraction of mergers with different $L / S_{h}$. The solid line in the upper of the two graphs is the fit using Eq. (11). Lower two graphs show the same as the upper graphs but now for the fraction $L / S_{s}$. Results are shown for progenitor halos of mass larger than $10^{12} M_{\odot}$ and mergers with mass ratio $M_{h} / M_{s} \leq 4$.

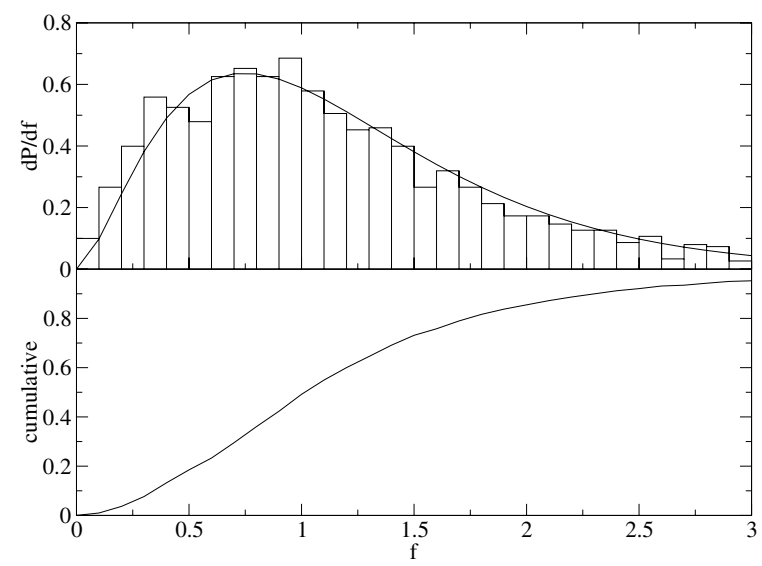

Fig. 20. Upper graph: distribution of parameter $f$ found in the simulations. Lower graph: corresponding cumulative fraction found in the simulation. Results are shown for progenitor halos of mass larger than $10^{12} M_{\odot}$ and mergers with mass ratio $M_{h} / M_{s} \leq 4$.

In particular in most cases the calculated pericenter distances are more than a factor two larger than those used generally in merger simulations. First results from simulations using larger pericenter distances indicate more isotropic remnants, which 
agree better with observations (Naab \& Burkert, in preparation). About half of the orbits are close to parabolic and those which are not show larger pericenter distances on average. The tight correlation $r_{\text {peri }} \propto \epsilon^{2.17}$ which we find between the pericenter distance and the circularity just supports that we have chosen halos when they are still well separated and not much interaction has taken place. Interaction leads to a circularization of the orbit predicting an increase of the circularity $\epsilon$ on average with decreasing pericenter distance $r_{\text {peri }}$. On the other hand hyperbolic orbits need to have very high kinetic energies to allow for small pericenter distances which the simulation shows to be unlikely. The pericenter distances however, show in the case of hyperbolic orbits a correlation with the impact parameter of the form $r_{\text {peri }} \propto b^{0.72}$, allowing to disentangle the different contributions to the orbital angular momentum using the additional correlation $L_{\mathrm{sp}} \propto r_{\text {peri }}^{0.53}$.

The distribution of circularities found is in contrast to what is commonly assumed in semi-analytic models of galaxy formation, which assume a random distribution of circularities for the orbits of infalling satellites. Instead our results indicate a distribution with a peak around $\epsilon \approx 0.5$.

We investigated the correlation between the spin plane of the main halo and the spin plane of the satellite with the orbital plane of the merger, finding no correlation. The planes are aligned randomly which gives statistical weight to certain merger geometries. Additionally the spin planes are not correlated with each other and the angle $i$ between the spin planes and the orbital plane shows no correlation with the other orbital parameters $r_{\text {peri }}$ and $e$ which justifies its use as an independent initial parameter. The pericentric argument $\omega$ follows a random distribution and shows no correlation with the other orbital parameters $r_{\text {peri }}$ and $e$.

We tested the requirement needed in the semi-analytic model of (Maller et al. 2002) for the acquiring of angular momentum of halos through major mergers. The authors define a parameter $f$ which should have on average a value of $f \sim 0.42$ to make their model reproduce the distributions found in simulation. However we find a mean value $f \sim 1$. which is larger than that required by their model, suggesting an inconsistency.

All presented results are equally valid for major mergers $\left(M_{1} / M_{2} \leq 4, M_{1} \geq M_{2}\right)$ and show no dependence on the mass ratio or mass of the merging objects supporting a self similar build up of structure. The use of the self-consistent initial conditions presented here will allow to test consistently the merger scenario for the formation of elliptical galaxies in the hierarchical universe without covering the whole parameter space and provides appropriate statistical weights for different initial conditions.

Acknowledgements. The authors would like to thank Thorsten Naab and Simon White for useful comments and Hugues Mathis for his help dealing with the simulation outputs. We would also like to thank Andrew Benson for intensive discussion and pointing out the importance of the Hubble flow for the eccentricities of orbits. S.K. acknowledges support by PPARC Theoretical Cosmology Rolling Grant.

\section{References}

Barnes, J. E. 1988, ApJ, 331, 699

Barnes, J. E., \& Hernquist, L. 1992, ARA\&A, 30, 705

Bender, R. 1988, AAP, 193, L7

Bender, R., Doebereiner, S., \& Moellenhoff, C. 1988, AAPS, 74, 385

Bendo, G. J., \& Barnes, J. E. 2000, MNRAS, 316, 315

Burkert, A., \& Naab, T. 2003a, preprint

[arXiv: astro-ph/0301385]

Burkert, A., \& Naab, T. 2003b, preprint

[arXiv: astro-ph/0305076]

Goldstein, H., Poole, C., \& Safko, J. 2002, Classical mechanics (3rd ed.) H. Goldstein, C. Poolo, \& J. Safko (San Francisco: AddisonWesley)

Hernquist, L. 1992, ApJ, 400, 460

Hernquist, L. 1993a, ApJS, 86, 389

Hernquist, L. 1993b, ApJ, 409, 548

Kauffmann, G., Colberg, J. M., Diaferio, A., \& White, S. D. M. 1999, MNRAS, 303, 188

Lemson, G., \& Kauffmann, G. 1999, MNRAS, 302, 111

Maller, A. H., Dekel, A., \& Somerville, R. 2002, MNRAS, 329, 423

Naab, T., Burkert, A., \& Hernquist, L. 1999, ApJ, 523, L133

Naab, T., \& Burkert, A. 2003, ApJ, 597, 893

Negroponte, J., \& White, S. D. M. 1983, MNRAS, 205, 1009

Peebles, P. J. E. 1993, Princeton Series in Physics (Princeton, NJ: Princeton University Press)

Porciani, C., Dekel, A., \& Hoffman, Y., 2002, MNRAS, 332, 325

Toomre, A., \& Toomre, J. 1972, ApJ, 178, 623 (TT72)

Tormen, G. 1997, MNRAS, 290, 411

Vitvitska, M., Klypin, A. A., Kravtsov, A. V., et al. 2002, ApJ, 581, 799 